\title{
Análisis con componentes principales y estimación de parámetros genéticos para medidas zoométricas en caballo pura raza española de México
}

Domínguez-Viveros, J. ${ }^{\circledR}$; Rodríguez-Almeida, F.A.; Burrola-Barraza, M.E.; Callejas-Juárez, N. y Ortega-Gutiérrez, J.A.

Facultad de Zootecnia y Ecología. Universidad Autónoma de Chihuahua. Chihuahua. México.

PALABRAS CLAVE ADICIONALES

Correlaciones genéticas.

Heredabilidad.

Mejoramiento genético.

Caracteres morfológicos.

Equinos.

\section{RESUMEN}

En el Caballo de Pura Raza Española (CPRE), los objetivos de selección son las disciplinas ecuestres en las que se desenvuelve, y los criterios de selección son las medidas zoométricas (MZ). El objetivo de este trabajo fue caracterizar el CPRE con análisis de componentes principales (ACP); estimar parámetros genéticos (PAG) para $M Z$, y los componentes generados con el ACP. Las $M Z$ analizadas en 1806 individuos (n) fueron: perímetro torácico (PT), perímetro de rodilla, perímetro de caña, longitud escápulo - isquial (LEI), anchura de pecho (AP), altura del hueco sub - esternal (AHS), altura de pecho (ALP), altura a la cruz. El ACP se realizó con la matriz de correlaciones y el software SAS. Para los PAG se realizaron dos análisis multivariados: con todas las MZ, y otro a partir del ACP. Con el soffware MTDFREML se estimó la heredabilidad ( $h^{2}$ ) para cada variable y las correlaciones genéticas ( $\mathrm{rg}$ ) entre variables. Del ACP, cuatro componentes se utilizaron para PAG. Del ACP, las variaciones en tamaño se atribuyen a PT y ALP, para conformación media a AHS; AP y LEI, definen caballos pequeños. Las $h 2$ para MZ oscilaron de 0.12 a 0.55 y un promedio de 0.31 ; todas las rg fueron positivas en el intervalo de 0.12 a 0.98 (promedio de 0.65 ). Las $h^{2}$ en los componentes fluctuaron de 0.29 a 0.47 (promedio de 0.38 ); las rg en su mayoría fueron positivas de 0.12 a 0.65 , y una negativa $(-0.13)$. En las $M Z$ la respuesta a la selección directa estará en función de $h^{2}$, y la posible respuesta correlacionada será por la magnitud y el signo de las rg. El ACP caracterizó al CPRE a través de tres estratos, y pueden ser uilizados en los criterios de selección.

\section{Principal components analysis and estimation of genetic parameters for zoometric measurements of purebred Spanish horses in Mexico}

\section{SUMMARY}

\section{ADDITIONAL KEYWORDS}

Genetic correlations.

Heritability.

Genetic improvement.

Morphological traits.

Equines.

\section{INFORMATION}

Cronología del artículo.

Recibido/Received: 01.04 .2018

Aceptado/Accepted: 20.05.2019

On-line: 15.07.2019

Correspondencia a los autores/Contact e-mail:

joeldguezviveros@yahoo.com.mx
In the Purebred Spanish Horse (PSH), the selection objectives are based on the equestrian disciplines in which this breed participates, while the selection criteria are the zoometric measurements (ZM). The objective of this paper was to characterize PSH using principal component analysis (PCA); and to estimate genetic parameters (GPA) for ZM, and the component generated by PCA. The ZM analyzed in 1806 individuals ( $\mathrm{n}$ ) were: thoracic perimeter (TP), knee perimeter, cannon perimeter, scapulo-ischial length (SIL), chest width (CW), height of the substernal space (HSS), chest height $(\mathrm{CH})$, height at the withers. The PCA was performed on the correlation matrix using the SAS soffware. For the GPA, two multivariate analyzes were carried out: with all the MZ, and another based on the PCA. The MTDFREML software was used to estimate the heritability $\left(\mathrm{h}^{2}\right)$ of each variable and the genetic correlations $(\mathrm{rg})$ between variables. Four components from the PCA were used to estimate the GPA. According to the PCA, the variations in size are defined by TP and CH, HSS defines medium horses, and CW and SIL define small horses. The h2 for $Z M$ ranged from 0.12 to 0.55 , with an average of 0.31 ; all $\mathrm{rg}$ were positive, in the range of 0.12 to 0.98 (average of 0.65 ). The $\mathrm{h}^{2}$ of the components fluctuated between 0.29 and 0.47 (average of $0.38)$; the rg were mostly positive, ranging from 0.12 to 0.65 , with one negative value $(-0.13)$. In the $\mathrm{ZM}$, the response to direct selection will be a function of $\mathrm{h} 2$, and the possible correlated response will be by the magnitude and sign of the rg. The PCA characterized the PSH across three levels that could be used as selection criteria.

\section{INTRODUCCIÓN}

La denominación como Caballo de Pura Raza Española (CPRE) fue adoptada en 1912, año en que se creó el libro genealógico en España (Varela et al., 2007); en México, el registro genealógico data de 1977 con información genealógica e integridad en los pedigrís que se remonta al año de 1905 (Domínguez-Viveros et al., 2012). En el mejoramiento genético del CPRE, los objetivos de selección están en función de las disciplinas ecuestres en las que se desenvuelve, tales como: 
doma, enganche, salto, alta escuela, rejoneo, gimnasia y prueba completa a través del campo. Los criterios de selección se desarrollan a partir de variables lineales y medidas zoométricas (MZ) considerados en el proceso de valoración para ingresar al registro de reproductores del libro genealógico de pureza de raza (SAGARPA, 2009; ANCCE, 2012).

La estructura, conformación y tamaño del caballo está en función de las MZ; las cuales varían de acuerdo con la disciplina ecuestre en la que se desenvuelve el individuo (Varela et al., 2009). La conformación del caballo, con la definición y disposición del cuerpo, define los límites del movimiento, funcionalidad y rendimiento (Sánchez-Guerrero et al., 2016); asimismo, el desempeño y rendimiento en las disciplinas ecuestres es el resultado de una combinación de las $\mathrm{MZ}$ con caracteres fisiológicos y de comportamiento, que pueden ser heredables (Saastamoinen y Barrey, 2000; Vostry et al., 2011). El tamaño y la conformación del cuerpo son rasgos importantes en todas las razas de caballos, y están sujetos a un estricto proceso de selección a través del tiempo (Brooks et al., 2010).

Con el análisis de componentes principales, se generan nuevas variables (componentes) que permiten explicar de la mejor manera las interrelaciones a través de las MZ (Flores et al., 2018), lo cual posibilita caracterizar el CPRE en función de las exigencias de las disciplinas ecuestres; y a su vez, las nuevas variables producto de la combinación lineal de las variables originales, pueden tener interpretación o aplicaciones para ser utilizadas en esquemas de mejoramiento genético a través de índices de selección (Kirkpatrick y Meyer, 2004; Karacaören y Kadarmideen, 2008; Vostry et al., 2012), así como para la caracterización morfoestructural de razas equinas (Salamanca et al., 2017). No obstante, para el diseño de estrategias de selección y cuantificar la respuesta a la selección, se requiere estimar los parámetros genéticos asociados a los criterios de selección (Arnason y Van Vleck, 2000; Vostry et al., 2011). Con base en lo anterior, los objetivos del presente estudio fueron: a) estimar parámetros genéticos para $\mathrm{MZ}$; b) caracterizar el CPRE con un análisis de componentes principales de las $\mathrm{MZ}$; y, c) estimar parámetros genéticos para las variables generadas con el análisis de componentes principales.

\section{MATERIALES Y MÉTODOS}

La base de datos estuvo conformada por 1806 equinos con datos de ocho MZ (media \pm desviación

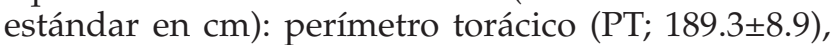
perímetro de la rodilla $(\mathrm{PR} ; 32.3 \pm 1.8)$, perímetro de caña (PC; 20.4 \pm 1.3 ), longitud escápulo - isquial (LEI; $160.7 \pm 5.9)$, anchura de pecho (AP; $43.1 \pm 3.0)$, altura del hueco sub - esternal (AHS; 83.1 \pm 5.1 ), altura de pecho

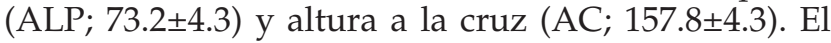
análisis de componentes principales (ACP) se realizó con base en la matriz de correlaciones de Pearson, utilizando el procedimiento para ACP del programa para análisis estadísticos SAS (SAS, 2005); posteriormente, con los eigenvectores producto de los componentes generados, se realizaron las transformaciones lineales para cada individuo a través de las ocho variables analizadas (Peña, 2002; Cuadras 2007).

Se realizaron dos análisis multivariados uno con las ocho MZ y otro a partir del ACP; los componentes que explicaron más del $90 \%$ de la variabilidad total se utilizaron para caracterizar el CPRE y para estimar parámetros genéticos. A partir del modelo mixto: $\mathrm{y}=$ $\mathrm{Xb}+\mathrm{Za}+\mathrm{e}$; donde $\mathrm{y}$ es el vector de registros; $\mathrm{b}$ es el vector de efectos fijos, que incluyó rancho, año, época de nacimiento y sexo, más la covariable de edad del individuo en función lineal y cuadrática; a es el vector de efectos aleatorios, genéticos aditivos directos; e es el vector de efectos residuales aleatorios; X y Z son matrices de incidencia que relacionan a las observaciones en y con los respectivos efectos. El arreglo matricial para el análisis multivariado quedo definido como:

$$
\left[\begin{array}{l}
y_{i} \\
y_{j}
\end{array}\right]=\left[\begin{array}{cc}
x_{i} & 0 \\
0 & x_{j}
\end{array}\right]\left[\begin{array}{l}
b_{i} \\
b_{j}
\end{array}\right]+\left[\begin{array}{cc}
z_{i} & 0 \\
0 & z_{j}
\end{array}\right]\left[\begin{array}{l}
a_{i} \\
a_{j}
\end{array}\right]+\left[\begin{array}{l}
e_{i} \\
e_{j}
\end{array}\right]
$$

La dimensión y estructura de las matrices X y Z estuvo en función del número de individuos $(\mathrm{N})$ con datos para cada variable analizada; las suposiciones del modelo fueron: $\mathrm{E}[\mathrm{y}]=\mathrm{X} \beta, \mathrm{E}[\mathrm{a}]=0 \mathrm{yE}[\mathrm{e}]=0$, y la estructura de varianzas y covarianzas quedo como:

$$
\operatorname{Var}\left[\begin{array}{l}
a_{i} \\
a_{j} \\
e_{i} \\
e_{j}
\end{array}\right]=\left[\begin{array}{cccc}
A \sigma_{a i}^{2} & A \sigma_{a i a j} & 0 & 0 \\
A \sigma_{a i a j} & A \sigma_{a j}^{2} & 0 & 0 \\
0 & 0 & I_{N} \sigma_{e i}^{2} \sigma_{e i e j} \\
0 & 0 & \sigma_{e i e j} I_{N} \sigma_{e j}^{2}
\end{array}\right]
$$

, donde: A, es la matriz con las relaciones genéticas aditivas (parentesco) entre todos los animales en el pedigrí; $\sigma_{a i}^{2}=$ varianzas de efectos genéticos aditivos directos; $\sigma_{\text {aiaj }}=$ covarianzas entre efectos genéticos aditivos directos; $\sigma_{\text {ei }}^{2}=$ varianzas residuales; $\sigma_{\text {eiej }}=$ covarianzas entre efectos residuales. El archivo de pedigrí estuvo conformado por 7498 ancestros nacidos a partir de 1977. Con el procedimiento de máxima verosimilitud restringida y libre de derivadas, se estimó las varianzas y covarianzas genéticas y fenotípicas; así como la heredabilidad $\left(\mathrm{h}^{2}\right)$ para cada variable, y las correlaciones genéticas $\left(r_{g}\right)$ a través de las variables de estudio; el análisis se realizó con el software MTDFREML (Boldman et al., 1995).

\section{RESULTADOS}

El coeficiente de variación a través de las MZ osciló de $2.72 \%(\mathrm{AC})$ a $6.96 \%(\mathrm{AP})$, con un valor promedio de $5.24 \%$; lo cual sugiere que la población analizada tiene cierto grado de uniformidad. Altos niveles de variación pueden indicar cierto grado de introgresión o adaptaciones a diferentes sistemas de cría dentro de una población (Flórez et al., 2018). Producto del ACP, en la Tabla I se presentan los ocho eigenvectores de los componentes generados; cuatro componentes (E1, E2, E3 y E4) explicaron el $94.8 \%$ de la varianza total, los cuales se utilizaron para describir el CPRE y estimar parámetros genéticos. En la Figura 1, se presentan tres 
Tabla I. Resultados del análisis de componentes principales, ocho eigenvectores $\left(\mathrm{E}_{\mathrm{i}}\right)$ para igual número de componentes (Principal component analysis results, eight eigenvectors (Ei) for equal number of components).

\begin{tabular}{|c|c|c|c|c|c|c|c|c|}
\hline Item $¥$ & E1 & E2 & E3 & $\mathrm{E} 4$ & E5 & E6 & E7 & E8 \\
\hline$A C$ & 0.2414 & 0.3816 & 0.0886 & 0.6498 & -0.1157 & -0.1439 & 0.0126 & -0.5759 \\
\hline LEI & 0.3390 & 0.2951 & 0.7616 & -0.4342 & -0.1714 & -0.0042 & -0.0057 & 0.0003 \\
\hline AP & 0.1659 & 0.1129 & 0.0738 & -0.0329 & 0.9406 & -0.2616 & -0.0025 & -0.0050 \\
\hline AHS & 0.0986 & 0.7364 & -0.3155 & 0.0597 & -0.0889 & -0.0471 & 0.0216 & 0.5782 \\
\hline ALP & 0.1411 & -0.3573 & 0.4030 & 0.5848 & -0.0227 & -0.1170 & -0.0070 & 0.5778 \\
\hline PT & 0.8736 & -0.2751 & -0.3712 & -0.1213 & -0.0883 & 0.0284 & -0.0102 & 0.0004 \\
\hline PR & 0.0699 & 0.0931 & 0.0711 & 0.1553 & 0.2139 & 0.8580 & -0.4185 & 0.0107 \\
\hline PC & 0.0400 & 0.0164 & 0.0429 & 0.0615 & 0.1027 & 0.3974 & 0.9078 & 0.0036 \\
\hline $\operatorname{Var}(\%)^{\dagger}$ & 48.7 & 22.6 & 16.3 & 7.2 & 3.2 & 1.2 & 0.6 & 0.2 \\
\hline
\end{tabular}

*Medidas zoométricas: $\mathrm{PT}=$ perímetro torácico; $\mathrm{PR}=$ perímetro de la rodilla; $\mathrm{PC}=$ perímetro de caña; $\mathrm{LEI}=$ longitud escápulo - isquial; $\mathrm{AP}=$ anchura de pecho; $\mathrm{AHS}=$ altura del hueco sub - esternal; $\mathrm{ALP}=$ altura de pecho; $\mathrm{AC}=$ altura a la cruz. ${ }^{\dagger} \mathrm{Var}(\%)=$ proporción de la varianza total, que es explicada por cada componente

agrupaciones o clasificaciones del CPRE en función de la dimensión y la conformación, graficando la relación del E1 con respecto a E2, E3 y E4. Las variaciones en cuanto al tamaño de los caballos se pueden atribuir al PT dado el signo negativo en los componentes 2 al 4 (Tabla I); además, asociado al signo en los componentes, las diferencias en los caballos de mayor tamaño se pueden atribuir a la ALP, para los caballos de conformación media las diferencias se pueden atribuir a la AHS; AP junto con LEI, definen la diferencia en los caballos más pequeños (Figura 1). Por otro lado, las $\mathrm{h}^{2}$ para las MZ fueron de mediana a alta magnitud (Tabla II), oscilaron de 0.12 a 0.55 y un valor promedio de 0.31 ; además, todas las $r_{g}$ fueron positivas en el intervalo de 0.12 a 0.98 , y un valor promedio de 0.65 . Las $\mathrm{h}^{2}$ en los componentes fluctuaron de 0.29 a 0.47 , y un promedio de 0.38; las $r_{g}$ en su mayoría fueron positivas en el intervalo de 0.12 a 0.65 , con excepción de la $\mathrm{r}_{\mathrm{g}}$ entre los componentes 3 y 4 que fue negativa de mediana magnitud (Tabla II).

\section{DISCUSIÓN}

Es estudios afines, Brooks et al. (2010) con base en el análisis de componentes principales en caracteres morfológicos de 65 razas de equinos, reportaron que los dos componentes de mayor impacto, dado la variabilidad que explicaban, estaban asociados al tamaño y la conformación de los caballos evaluados. Cervantes et al. (2009), con el ACP en caballo hispano árabe, reportaron que el primer factor explicó un $21.4 \%$ de la

Tabla II. Parámetros genéticos ${ }^{\dagger}$ para medidas zoométricas y componentes principales en Caballos de Pura Raza Español (Genetic parameters for zoometric measurements and main components in Spanish Pure Breed Horses).

\begin{tabular}{|c|c|c|c|c|c|c|c|c|}
\hline Ítem ${ }^{¥-\pi}$ & $A C$ & AHS & ALP & AP & LEI & $P C$ & PR & PT \\
\hline$A C$ & 0.55 & & & & & & & \\
\hline AHS & 0.91 & 0.19 & & & & & & \\
\hline ALP & 0.83 & 0.51 & 0.12 & & & & & \\
\hline AP & 0.73 & 0.86 & 0.24 & 0.25 & & & & \\
\hline LEI & 0.59 & 0.46 & 0.79 & 0.49 & 0.55 & & & \\
\hline PC & 0.56 & 0.12 & 0.86 & 0.82 & 0.83 & 0.16 & & \\
\hline PR & 0.78 & 0.65 & 0.66 & 0.85 & 0.71 & 0.98 & 0.33 & \\
\hline \multirow[t]{2}{*}{ PT } & 0.41 & 0.20 & 0.75 & 0.65 & 0.41 & 0.83 & 0.80 & 0.34 \\
\hline & C1 & $\mathrm{C} 2$ & $\mathrm{C} 3$ & $\mathrm{C} 4$ & & & & \\
\hline $\mathrm{C} 1$ & 0.47 & & & & & & & \\
\hline $\mathrm{C} 2$ & 0.30 & 0.29 & & & & & & \\
\hline $\mathrm{C} 3$ & 0.29 & 0.65 & 0.39 & & & & & \\
\hline C4 & 0.12 & 0.49 & -0.13 & 0.38 & & & & \\
\hline
\end{tabular}




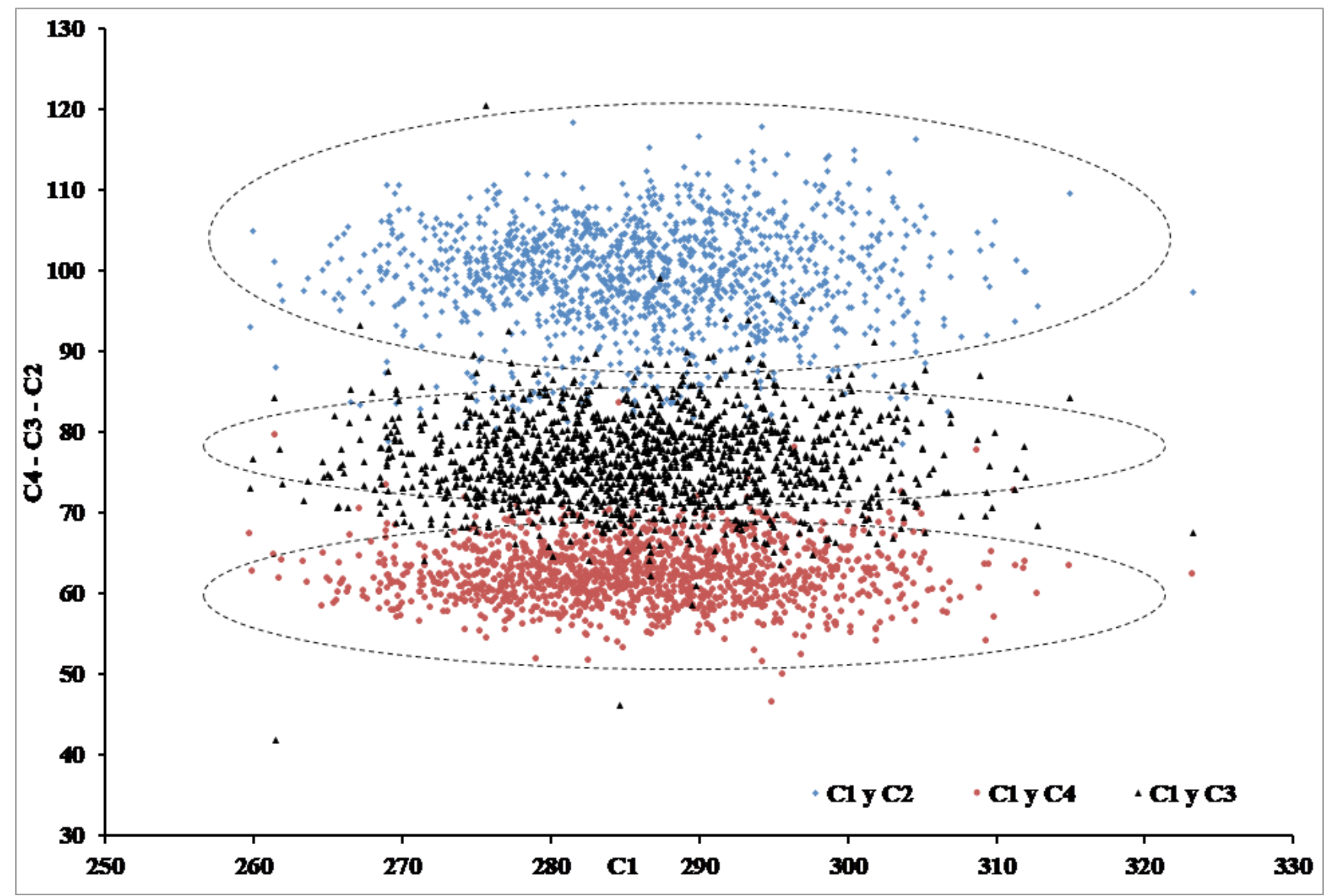

Figura 1. Caracterización del Caballo de Pura Raza Española a través de los cuatro componentes (C1, C2, C3 y C4) seleccionados (Characterization of the Spanish Purebred Horse through the four components (C1, C2, C3 and C4) selected).

varianza total y estaba asociado a rasgos de altura, los cuales determinan el tamaño general del caballo; un segundo factor que explicó el $8.5 \%$ de la varianza, se relacionó a variables torácicas. El ACP permite identificar grupos de animales de conformación corporal similar y determinar su orientación zootécnica (Salamanca et al., 2017).

En los cambios a través del tiempo, el CPRE ha tenido un ligero aumento en la talla; sin embargo, los mayores cambios se han observado en la longitud corporal modificando la proporcionalidad corporal de la raza, de medio línea a sub longilínea (Varela et al., 2007). Los análisis de MZ permiten cuantificar las dimensiones del patrón racial y caracterizar fenotípicamente las subpoblaciones dentro de razas, como es el caso del CPRE y su relación con la población de origen u otras subpoblaciones en los países donde se produce; la Figura 1 presenta las dimensiones y clasificación del CPRE en México. Los caracteres AP, PT y LEI que definen la conformación del individuo, están asociados al motor o rendimiento de los caballos; AHS, ALP, y AC junto con otras mediciones de cabeza y cuello contribuyen a la forma y movimiento de los caballos; y, PC y PR se relacionan con la fortaleza requerida a través de las disciplinas ecuestres (Peña et al., 2009; Sobczuk y Komosa, 2012). Sánchez et al. (2013) analizando las relaciones genéticas entre las MZ y las variables de trote, movimientos y doma, reportaron la importancia de PT, PR, PC y AC en esas disciplinas. Dado la conformación y tamaño de los équidos, existen marcadas diferencias a través de razas para variables de funcionalidad, el Caballo Pura Sangre Ingles es característico para el galope; para el trote, el Trotador Español, Alemán y Frances; para silla, el CPRE ha presentado las características idóneas como importante exponente; para el salto, el caballo Hannoveriano; y para raid, el caballo Árabe (Varela et al., 2009).

Con base en las $\mathrm{h}^{2}$ las ocho MZ presentan variabilidad genética, un siguiente paso es analizar la magnitud y el sentido de la posible respuesta a la selección; conjuntamente, dado la magnitud y el signo de las $r_{g}$ se esperaría una respuesta asociada o correlacionada a través de todas las MZ, la cual debe ser valorada bajo diferentes esquemas de selección. En otras poblaciones de CPRE, evaluando las mismas MZ del presente estudio, han reportado $\mathrm{h}^{2}$ superiores $\mathrm{y}_{\mathrm{g}}$ menores: Molina et al. (1999) publicaron estimaciones de $h^{2} \mathrm{y} \mathrm{r}_{\mathrm{g}}$ en los intervalos de 0.35 a 0.95 y 0.11 a 0.94 , con promedios de 0.59 y 0.46 , respectivamente; Gómez et al. (2008) para $\mathrm{h}^{2} \mathrm{y} \mathrm{r}_{\mathrm{g}}$ obtuvieron promedios de 0.36 (0.25 a 0.57) y 0.62 (0.35 a 0.88), respectivamente; Sánchez-Guerrero et al. (2016) las $\mathrm{h}^{2} \mathrm{y} \mathrm{r}_{\mathrm{g}}$ oscilaron de 0.39 a 0.80 , y de 0.25 a 0.90, respectivamente. En otras razas de equinos, Sole et al. (2014) analizando trece MZ en caballo Menorca, obtuvieron una $\mathrm{h}^{2}$ promedio de $0.35 \mathrm{y}$ valores máximos de 0.75; Bakhtiari y Heshmat (2009), analizando diez 
variables en caballo Pura Sangre Iraní, reportaron heredabilidades en el intervalo de 0.22 a 0.49 , así como todas las correlaciones genéticas positivas de 0.18 a 0.92 .

Heredabilidades superiores a 0.40 se pueden atribuir a problemas de análisis sobre estimaciones utilizando modelos inadecuados o incompletos. En MZ asociadas al crecimiento corporal, los efectos genéticos maternos y las correlaciones genéticas con los efectos genéticos directos deben ser consideradas en los modelos estadísticos (Torzynski et al., 2005); en el presente estudio no se incluyeron los efectos genéticos maternos, debido a que en el desarrollo del análisis multivariado la estructura de (co)varianzas de los efectos genéticos maternos presentó estimaciones con tendencia a cero. Por otro lado, el que todas las correlaciones genéticas sean positivas, de mediana a alta magnitud, puede tener repercusiones en el diseño de programas de mejoramiento genético; cada disciplina ecuestre requiere de caballos con características propias, y los criterios de selección con base en las combinaciones de las MZ son diferentes a través de disciplinas.

Para implementar programas de mejoramiento genético con base en la selección de múltiples características, se requiere el diseño de índices de selección, donde se examine las relaciones genéticas y fenotípicas entre los criterios y los objetivos de selección, así como los valores ponderales y/o porcentuales de las variables implicadas (Arnason y Van Vleck, 2000; Sánchez-Guerrero et al., 2017). Las aplicaciones del ACP en genética cuantitativa se contemplan en reducir la dimensión y arreglo de la matriz de varianzas y covarianzas, asociado a los requerimientos de cómputo y estructura de la base de datos, y en algunas condiciones el ACP reduce un análisis multivariado a una serie de análisis univariados; además de visualizar e identificar ciertos patrones de variación genética (Kirkpatrick y Meyer, 2004; Vostry et al., 2012). El ACP es una técnica multivariada que permite examinar la relación existente entre los criterios de selección y está asociado a las propiedades, usos y aplicaciones de un índice de selección (Godshalk y Timothy, 1988; Ruales-España y Manrique, 2007). Con estos resultados, se esperaría una respuesta a la selección al utilizar los componentes principales, con objetivos de selección en función del tamaño y la conformación requeridos en las disciplinas ecuestres.

\section{CONCLUSIONES}

Las medidas zoométricas evaluadas presentaron heredabilidades de mediana a alta magnitud, y al considerarlas en los esquemas de selección se esperará una respuesta a la selección importante; conjuntamente, todas las correlaciones genéticas fueron positivas, lo que implica posibles respuestas a la selección correlacionadas en el mismo sentido y posibilita una selección sobre distintas funcionalidades en la misma raza, sin esperanza de grandes antagonismos.

El análisis de componentes principales caracterizó morfológicamente al caballo de pura raza española en México a través de tres estratos o niveles, en función del tamaño y la conformación.

\section{AGRADECIMIENTOS}

Se agradece a la Asociación Nacional de Criadores de Caballos de Pura Raza Española por proporcionar la base de datos analizada; en el marco del convenio de colaboración con la Universidad Autónoma de Chihuahua y el Consejo Nacional de los Recursos Genéticos Pecuarios.

\section{BIBLIOGRAFÍA}

ANCCE 2012, 'Certificado de aptitud básica para la reproducción. Libro Genealógico del Caballo de Pura Raza Española', Asociación Nacional de Criadores de Caballos de Pura Raza Española. 10 p.

Arnason, T \& Van Vleck, LD 2000, 'Genetic improvement of the horse', In: The genetic of horse, CABI Publishing. Cambridge, MA, USA. pp. 473-497.

Bakhtiari, J \& Heshmat, G 2009, 'Estimation of genetic parameters of conformation traits in Iranian Thoroughbred horses', Livestock Science, vol. 123, pp. 116-120.

Boldman, KG, Kriese, LA, Van Vleck, LD, Van Tassell, CP \& Kachman, SD 1995, 'A Manual for use of MTDFREML. A set of programs to obtain estimates of variances and covariances (Draft)', USDA. ARS. $114 \mathrm{p}$.

Brooks, SA, Makvandi-Nejad, S, Chu, E, Allen, JJ, Streeter, C, Gu, E, McCleery, B, Murphy, BA, Bellone, R \& Sutter, NB 2010, 'Morphological variation in the horse: defining complex traits of body size and shape', Animal Genetics, vol. 41, pp. 159-165.

Cervantes, I, Baumung, R, Molina, A, Druml, T, Gutiérrez, JP, Solkner, J \& Varela, M 2009, 'Size and shape analysis of morpho functional traits in the Spanish Arab horse', Livestock Science, vol. 125, pp. 43-49.

Cuadras, CM 2007, 'Nuevos métodos de análisis multivariante', CM Ceditions. Barcelona, España. $150 \mathrm{p}$.

Domínguez-Viveros, J, Rodríguez-Almeida, FA, Ortega-Gutiérrez, JA \& Santellano-Estrada, E 2012, 'Análisis de la información genealógica y estimación de parámetros de poblaciones en bovinos de Lidia y equinos de Pura Raza Española de México', Revista Científica FCVLUZ, vol. 22, pp. 443-450.

Flórez, JM, Hernández, M de J, Bustamante, M de J \& Vergara OD 2018, 'Caracterización morfoestructural de tres poblaciones de ovino de pelo criollo colombiano "OPC" ' Archivos de Zootecnia, vol. 67, pp 340-348.

Godshalk, EB \& Timothy, DH 1988, 'Factor and principal component analyses as alternatives to index selection', Theoretical and Applied Genetics, vol. 76, pp. 352-360.

Gómez, MD, Varela, M, Molina, A \& Goyache, F 2008, 'Efecto semental $x$ ganadería en la estimación de parámetros genéticos en caracteres de tamaño en el caballo Pura Raza Española: resultados preliminares', Información Técnica Económica Agraria, vol. 104, pp. 256-261.

Karacaön, B \& Kadarmideen, HN 2008, 'Principal components and clustering analysis of functional traits in Swiss dairy cattle', Turkish Journal of Veterinary and Animal Sciences, vol. 32, pp. 163-171.

Kirkpatrick, M \& Meyer, K 2004, 'Direct estimation of genetic principal components: simplified analysis of complex phenotypes', Genetics, vol. 168, pp. 2295-2306.

Molina, A, Varela, M, dos Santos, R \& Rodero, A 1999, 'Genetic parameters of morpho functional traits in Andalusian horse', Livestock Production Science, vol. 60, pp. 295-303.

Peña, D 2002, Análisis de datos multivariantes. Editorial McGraw Hill. Madrid, España. 400 p.

Peña, F, Gómez, MD, Bartolomé, E \& Varela, M 2009, 'Valoración morfológica en équidos', En: Valoración morfológica de los animales domésticos. Sociedad Española de Zooetnólogos, Madrid, España. pp. 203-229.

Ruales-España, FR \& Manrique, PC 2007, 'Use of principal components análisis for building up a production-type index for Romosinuano (Bos taurus) cattle', Revista Colombiana de Ciencias Pecuarias, vol. 20, pp. 124-128. 
Saastamoinen MT \& Barrey, E 2000, 'Genetics of conformation, locomotion and physiological traits', In: The genetic of horse, CABI Publishing. Cambridge, MA, USA. pp. 439-472.

SAGARPA 2009, 'Reglamento técnico de la Asociación Nacional de Criadores de Caballos de Pura Raza Española', Secretaria de Agricultura, Ganadería, Desarrollo Rural, Pesca y Alimentación. 65 p.

Salamanca, CA, Parés-Casanova, PM, Crosby, RA \& Monroy N 2017, 'Análisis biométrico del caballo criollo Araucano', Archivos de Zootecnia, vol. 66, pp 107-112.

Sánchez, MJ, Gómez, MD, Peña, F, García, MJ, Morales, JL, Molina , A \& Varela, M 2013, 'Relations between conformation trait and gait characteristics in Pura Raza Español horse', Archives Animal Breeding, vol. 56, pp. 137-148.

Sánchez-Guerrero, MJ, Molina, A, Gómez, MD, Peña, F \& Varela, M 2016, 'Relationship between morphology and performance: signature of mass-selection in Pura Raza Español horse', Livestock Science, vol. 185, pp. 148-155.

Sánchez-Guerrero, MJ, Cervantes, I, Molina, A, Gutiérrez, JP \& Varela, M 2017, 'Designing an early selection morphological linear traits index for dressage in the Pura Raza Español horse', Animal, vol. 11 , pp. 948-957.

SAS 2005, SAS/STAT User's Guide (Release 9.0), Cary NC, USA: SAS Inst. Inc.

Sobczuk, D \& Komosa, M 2012, 'Morphological differentiation of polish Arabian horses - multivariate analysis', Bulletin of the Veterinary Institute in Pulawy, vol. 56, pp. 623-629.
Sole, M, Cervantes, I, Gutiérrez, JP, Gómez, MD \& Varela, M 2014, 'Estimation of genetic parameters for morphological and functional traits in Menorca horse', Spanish Journal of Agricultural Research, vol. 12, pp. 125-132.

Torzynski, G, Wolc, A \& Szwaczkowski, T 2005, 'Maternal effects on the conformation traits in half-bred horses', Journal of Animal Feed Science, vol. 14, pp. 595-605.

Varela, CM, Peña, BF, \& Gómez, MD 2007, 'El caballo de pura raza español', En: Las razas ganaderas de Andalucía; patrimonio ganadero andaluz, España. pp. 475-510.

Varela, M, Gómez, MD, Cervantes, I \& Peña, F 2009, 'Morfología y funcionalidad en los équidos', En: Valoración morfológica de los animales domésticos. Sociedad Española de Zooetnólogos, Madrid, España. pp. 701-727.

Vostry, L, Capkova, Z, Pribyl, J \& Mach, K 201 1, 'Analysis of Czech coldblooded horses: genetic parameters, breeding value and the influence of inbreeding depression on linear description of conformation and type characters', Czech Journal of Animal Science, vol. 56, pp. 217-230.

Vostry, L, Pribyl, J \& Simecek, P 2012, 'Reduction of trait for genetic evaluation of linear described traits in the Old Kladruber horse', Czech Journal of Animal Science, vol. 57, pp. 160-170. 